\title{
On the complexity of vertex-coloring edge-weightings
}

\author{
Andrzej Dudek 1 " and David Wajc ‘非 \\ ${ }^{1}$ Department of Mathematics, Western Michigan University, Kalamazoo, MI 49008, USA \\ ${ }^{2}$ Computer Science Department, Technion Israel Institute of Technology, Haifa 32000, Israel
}

received $18^{\text {th }}$ March 2010, revised $24^{\text {th }}$ February 2011, accepted $26^{\text {th }}$ October 2011.

Given a graph $G=(V, E)$ and a weight function $w: E \rightarrow \mathbb{R}$, a coloring of vertices of $G$, induced by $w$, is defined by $\chi_{w}(v)=\sum_{e \ni v} w(e)$ for all $v \in V$. In this paper, we show that determining whether a particular graph has a weighting of the edges from $\{1,2\}$ that induces a proper vertex coloring is NP-complete.

Keywords: vertex-coloring, 1-2-3 conjecture, NP-completeness

\section{Introduction}

For a given graph $G=(V, E)$, let $w: E \rightarrow \mathbb{R}$ be a weight function. We say that $w$ is proper if the coloring of the vertices $\chi_{w}(v)=\sum_{e \ni v} w(e), v \in V$, is proper. In 2004, Karoński, Łuczak, and Thomason (2004) showed that any graph with no components isomorphic to $K_{2}$ has a proper weighting from a finite set of reals. Furthermore, they conjectured that every graph with no components isomorphic to $K_{2}$ has a proper weighting from $W=\{1,2,3\}$. Addario-Berry, Dalal, McDiarmid, Reed, and Thomason (2007) showed that the above holds if $W=\{1, \ldots, 30\}$. This result was improved by Addario-Berry, Dalal, and Reed (2008), who showed that one can take $W=\{1, \ldots, 16\}$. Subsequently, Wang and Yu|(2008) proved that $W=\{1, \ldots, 13\}$ suffices. A recent breakthrough by Kalkowski, Karoński, and Pfender (2010) showed that the set of weights can be as small as $W=\{1,2,3,4,5\}$.

On the other hand, Addario-Berry, Dalal, and Reed (2008) showed that almost all graphs have a proper weighting from $\{1,2\}$. In this paper, we show that determining whether a particular graph has a proper weighting of the edges from $\{1,2\}$ is NP-complete. Consequently, there is no simple characterization of graphs with proper weightings from $\{1,2\}$, unless $\mathrm{P}=\mathrm{NP}$. Formally, let

$$
1-2 \text { WEIGHT }=\{G: G \text { is a graph having a proper weighting from }\{1,2\}\} .
$$

\footnotetext{
†Email: andrzej.dudek@wmich.edu.

‡Email: sdavidwa@cs.technion.ac.il This work was performed while the author was visiting Carnegie Mellon University.

1365-8050 @ 2011 Discrete Mathematics and Theoretical Computer Science (DMTCS), Nancy, France
} 


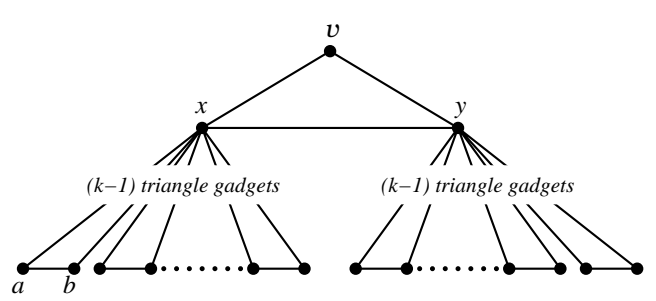

(a)

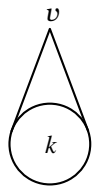

(b)

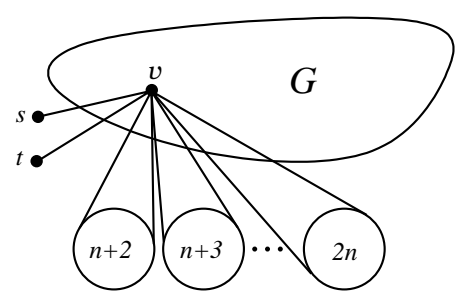

(c)

Fig. 1: A $k$-disallowing gadget (a) and its symbolic representation (b); a construction of $h(G)$ (c).

\section{Theorem 1.1 1-2WEIGHT is NP-complete.}

Before we prove this statement, we consider a similar theorem with a somewhat simpler proof, which we use as a template to prove Theorem 1.1. By analogy to 1-2WEIGHT we denote by $0-1$ WEIGHT the family of graphs with a proper weighting from $\{0,1\}$ and show the following:

Theorem 1.2 0-1WEIGHT is NP-complete.

\section{0-1WEIGHT is NP-complete}

Here we prove Theorem 1.2

First note that 0 -1WEIGHT is clearly in NP, since one can verify in polynomial time for a given graph whether a weighting of its edges from $\{0,1\}$ is proper.

Next we consider the well-known NP-hard problem

$$
\text { 3-COLOR }=\{G: G \text { is a graph having a proper 3-vertex-coloring }\} \text {. }
$$

In order to prove that 0-1WEIGHT is NP-hard (and hence NP-complete), we show a reduction from 3COLOR to 0-1WEIGHT. To this end, we define a polynomial time reduction $h$, such that $G \in 3$-COLOR if and only if $h(G) \in 0$-1WEIGHT. To achieve this, we need two auxiliary gadgets.

We refer to the first gadget as a triangle gadget. This consists of a triangle $x a b$, with $x$ referred to as the top and with $a$ and $b$ each having no other coinciding edges. Note that any proper weighting $w$ from $\{0,1\}$ of a graph with such a triangle must hold $w(x a) \neq w(x b)$; otherwise $\chi_{w}(a)=w(a b)+w(a x)=$ $w(b a)+w(b x)=\chi_{w}(b)$. Hence, $\{w(x a), w(x b)\}=\{0,1\}$ and so every such triangle gadget contributes exactly 1 to $\chi_{w}(x)$.

The second gadget, called a $k$-disallowing gadget, consists of a main triangle $v x y$ with $v$ referred to as the root and with $x$ and $y$ each constituting the top of $k-1$ distinct triangle gadgets (see Figure 1(a)). Note that in any proper weighting $w$ from $\{0,1\}, w(v x) \neq w(v y)$; otherwise, as both $\chi_{w}(x)$ and $\chi_{w}(y)$ have $k-1$ contributed by $x$ and $y$ 's triangles, $\chi_{w}(x)=w(x v)+w(x y)+k-1=w(y v)+w(y x)+$ $k-1=\chi_{w}(y)$. Therefore, if $w(x y)=0$ then $\left\{\chi_{w}(x), \chi_{w}(y)\right\}=\{k-1, k\}$ and, if $w(x y)=1$ then $\left\{\chi_{w}(x), \chi_{w}(y)\right\}=\{k, k+1\}$. In either case, $v$ has one neighbor $z \in\{x, y\}$ with $\chi_{w}(z)=k$, and consequently, $\chi_{w}(v) \neq k$ in any proper weighting from $\{0,1\}$. Also $\{w(v x), w(v y)\}=\{0,1\}$ and hence this gadget contributes exactly 1 to $\chi_{w}(v)$. 
Now we are ready to show a reduction from 3-COLOR to 0-1WEIGHT, $h$, such that $G \in 3$-COLOR if and only if $h(G) \in 0$-1WEIGHT. Let $G=(V, E)$ be a graph of order $n$. We may assume that $n \geq 3$. Otherwise, $n \leq 2$ and $G$ is in 3-COLOR and so it suffices to take as $h(G)$ an empty graph which is trivially in 0-1WEIGHT. For $n \geq 3$ we construct the graph $h(G)=(W, F)$ as follows (see Figure 1(c)). We start with $G=(V, E)$. For each $v \in V$ :

(i) connect $v$ to two new vertices, $s$ and $t$ (distinct for each $v$ );

(ii) add $n-1$ new $k$-disallowing gadgets for all $k \in\{n+2, n+3, \ldots, 2 n\}$ with $v$ as their root.

Clearly, $h(G)$ can be calculated in time polynomial in the size of $G$.

Fact 2.1 In $h(G)$ the following holds: any proper weighting $w$ from $\{0,1\}$ satisfies $\chi_{w}(v) \in\{n-$ $1, n, n+1\}$ for every $v \in V$.

Proof: Fix $v \in V$. Since $w(v s)+w(v t) \in\{0,1,2\}, v$ is the endpoint of $\operatorname{deg}(v) \leq n-1$ edges in $V$, and $v$ is the root of $(n-1) k$-disallowing gadgets (each contributing 1 to $\chi_{w}(v)$ ), we have:

$$
\chi_{w}(v) \in\{0,1,2\}+\{0,1, \ldots, \operatorname{deg}(v)\}+\{n-1\} \subseteq\{n-1, n, \ldots, 2 n\},
$$

where by $A+B$ we mean the set of all sums of an element from $A$ with an element from $B$. Observing the above and the fact that $v$ is the root of $k$-disallowing gadgets for all $k \in\{n+2, \ldots, 2 n\}$, we find that any proper weighting $w$ from $\{0,1\}$ satisfies $\chi_{w}(v) \in\{n-1, n, n+1\}$, as claimed.

It remains to show that $G \in 3$-COLOR if and only if $h(G) \in 0$-1WEIGHT.

First let us assume that $G \in 3$-COLOR. That means there exists a proper 3-coloring of $G$, say $\chi: V \rightarrow$ $\{n-1, n, n+1\}$. We define a weighting of the edges of $h(G), w: F \rightarrow\{0,1\}$ as follows. For all $e \in E$ let $w(e)=0$. For all $v \in V$, if $\chi(v)=n-1$ then $w(v s)=w(v t)=0$; otherwise, if $\chi(v)=n$ then $w(v s)=1$ and $w(v t)=0$; and finally, if $\chi(v)=n+1$ then $w(v s)=w(v t)=1$. All other edges (parts of gadgets) are weighted as follows: For a triangle gadget $x a b$ with $\operatorname{root} x, w(x a)=1, w(x b)=w(a b)=0$. For a $k$-disallowing gadget with root $v$, and main triangle $v x y, w(v x)=w(x y)=1, w(v y)=0$, and the weighting of all other triangle gadgets as described above. Note that $w$ is a proper weighting of $h(G)$ (satisfying $\chi_{w}(v)=\chi(v)$ for all $v \in V$ ), as required.

Now let us assume that $G \notin 3$-COLOR. Therefore, for all $\chi: V \rightarrow\{n-1, n, n+1\}, \chi$ is not proper. But, from Fact 2.1. any proper weighting from $\{0,1\}$ of $h(G)$ satisfies $\chi_{w}(v) \in\{n-1, n, n+1\}$ for all $v \in V$. Thus, there is no such proper weighting and hence $h(G) \notin 0$-1WEIGHT.

This completes the proof of Theorem 1.2

\section{1-2WEIGHT is NP-complete}

The proof of Theorem 1.1 extends the ideas introduced in the proof of Theorem 1.2 Since clearly 12WEIGHT is in NP, it remains to show that 1-2WEIGHT is NP-hard. As before, we show a reduction from 3-COLOR to 1-2WEIGHT. To this end, we define a polynomial time reduction $f$, such that $G \in$ $3-C O L O R$ if and only if $f(G) \in 1$-2WEIGHT. Below we define auxiliary gadgets.

As in Section 2, we will use a triangle gadget. Now note that every triangle $x a b$, with only $x$ having other adjacent edges ( $x$ is referred to as the top), contributes exactly 3 to $\chi_{w}(x)$ in any proper weighting $w$ from $\{1,2\}$. 


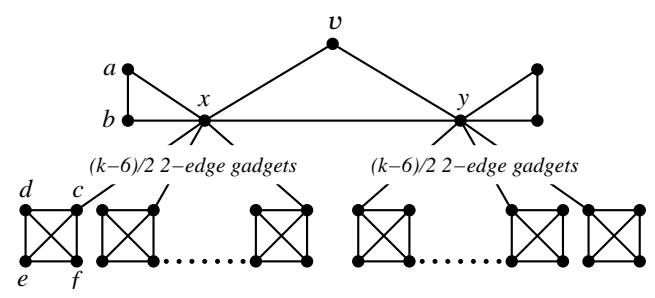

(a)

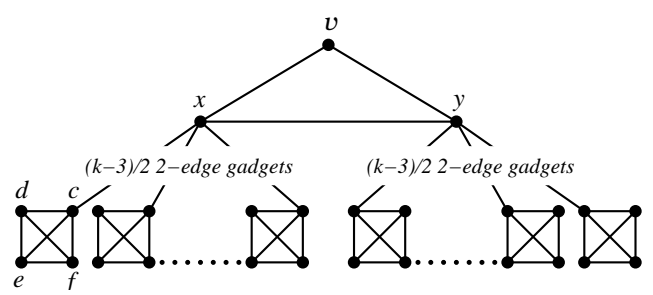

(b)

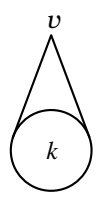

(c)

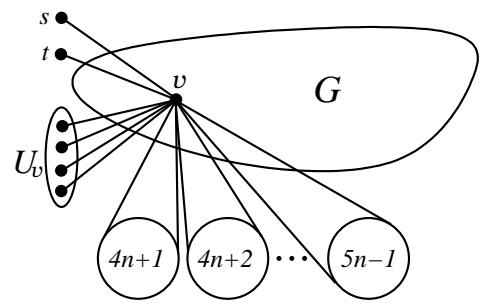

(d)

Fig. 2: A $k$-disallowing gadget (a) for even $k$, (b) for odd $k$ and their symbolic representation (c); a construction of $f(G),(\mathrm{d})$.

Now we define a 2-edge gadget consisting of the set of vertices $\{x, c, d, e, f\}$ with $x$ and $c$ adjacent and $\{c, d, e, f\}$ spanning a complete graph $K_{4}$. One can check that every proper weighting $w$ from $\{1,2\}$ of a graph adjacent to such a gadget only at $x$ requires $w(x c)=2$. We refer to $x$ as the endpoint.

We use the above gadgets to construct another gadget, called a $k$-disallowing gadget. As we will see, this gadget has similar properties as its namesake in Section 2. We therefore allow ourselves the re-use of the name for this new, slightly different, gadget. We assume that $k \geq 8$. The $k$-disallowing gadget contains a main triangle vxy with $v$ referred to as the root. Moreover, if $k$ is even, $x$ and $y$ each form the endpoint of $(k-6) / 2$ edge disjoint 2-edge gadgets and $x$ and $y$ are each tops of distinct triangle gadgets (see Figure 2(a) $)$. If $k$ is odd, $x$ and $y$ each form the endpoint of $(k-3) / 2$ edge disjoint 2-edge gadgets (see Figure 2(b) . Note that in any proper weighting $w$ from $\{1,2\}, w(v x) \neq w(v y)$; otherwise, since the weight contributed by gadgets to $\chi_{w}(x)$ and $\chi_{w}(y)$ is $k-3$, then $\chi_{w}(x)=w(x v)+w(x y)+k-3=$ $w(y v)+w(y x)+k-3=\chi_{w}(y)$. Therefore, for any $k$, if $w(x y)=1$ then $\left\{\chi_{w}(x), \chi_{w}(y)\right\}=\{k-1, k\}$ and, if $w(x y)=2$ then $\left\{\chi_{w}(x), \chi_{w}(y)\right\}=\{k, k+1\}$. In either case, $v$ has one neighbor $z \in\{x, y\}$ with $\chi_{w}(z)=k$, and consequently, $\chi_{w}(v) \neq k$ in any proper weighting from $\{1,2\}$. Also $\{w(v x), w(v y)\}=$ $\{1,2\}$, and hence this gadget contributes exactly 3 to $\chi_{w}(v)$.

Now we are ready to show a polynomial time reduction from 3-COLOR to 1-2WEIGHT, $f$, such that $G \in 3$-COLOR if and only if $f(G) \in 1$-2WEIGHT. Let $G=(V, E)$ be a graph of order $n$. As in Section 2, we may assume that $n \geq 3$. We construct the graph $f(G)=(W, F)$ as follows (see Figure 2(d)). We start with $G=(V, E)$. For each $v \in V$ :

(i) connect $v$ to two new vertices $s$ and $t$ (distinct for each $v$ );

(ii) connect $v$ to all vertices from a new set $U_{v}$ (distinct for each $v$ ) with $\left|U_{v}\right|=n-1-\operatorname{deg}(v)$; 
(iii) add $n-1$ new $k$-disallowing gadgets for all $k \in\{4 n+1,4 n+2, \ldots, 5 n-1\}$ with $v$ as their root. Clearly, $f(G)$ can be calculated in time polynomial in the size of $G$.

Fact 3.1 In $f(G)$ the following holds: any proper weighting $w$ from $\{1,2\}$ satisfies $\chi_{w}(v) \in\{4 n-$ $2,4 n-1,4 n\}$ for every $v \in V$.

Proof: Fix $v \in V$. Since $w(v s)+w(v t) \in\{2,3,4\}, v$ is the endpoint of $n-1$ edges with endpoints in $V \cup U_{v}$ and $v$ is the root of $(n-1) k$-disallowing gadgets (each contributing 3 to $\chi_{w}(v)$ ), we have:

$$
\chi_{w}(v) \in\{2,3,4\}+\{n-1, \ldots, 2 n-2\}+\{3 n-3\}=\{4 n-2, \ldots, 5 n-1\} .
$$

Observing the above and the fact that $v$ is the root of $k$-disallowing gadgets for all $k \in\{4 n+1,4 n+$ $2, \ldots, 5 n-1\}$, we find that any proper weighting $w$ from $\{1,2\}$ satisfies $\chi_{w}(v) \in\{4 n-2,4 n-1,4 n\}$, as claimed.

Now we show that $G \in 3$-COLOR if and only if $f(G) \in 1$-2WEIGHT.

First let us assume that $G \in 3$-COLOR. That means there exists a proper 3-coloring of $G$, say $\chi$ : $V \rightarrow\{4 n-2,4 n-1,4 n\}$. We define a weighting of the edges of $f(G), w: F \rightarrow\{1,2\}$ as follows. For all $e \in E$ let $w(e)=1$. For all edges $e=v u$ with $v \in V$ and $u \in U_{v}$ we set $w(e)=1$. For all $v \in V$, if $\chi(v)=4 n-2$ then $w(v s)=w(v t)=1$; otherwise, if $\chi(v)=4 n-1$ then $w(v s)=1$ and $w(v t)=2$; finally, if $\chi(v)=4 n$ then $w(v s)=w(v t)=2$. All other edges (parts of gadgets) are weighted as follows: For a triangle gadget $x a b$ with root $x, w(x a)=2, w(x b)=w(a b)=1$. For a 2-gadget defined by $\{x, c, d, e, f\}$ with $x$ adjacent to $c$, we have $w(x c)=w(c d)=w(c e)=w(d e)=$ $w(d f)=2$ and $w(c f)=w(e f)=1$. For a $k$-disallowing gadget with root $v$ and main triangle $v x y$, $w(v x)=w(x y)=2, w(v y)=1$, and the weighting of all other gadgets as described above. Note that $w$ is a proper weighting of $f(G)$ (satisfying $\chi_{w}(v)=\chi(v)$ for all $v \in V$ ), as required.

Next let us assume that $G \notin 3$-COLOR. Therefore, for all $\chi: V \rightarrow\{4 n-2,4 n-1,4 n\}, \chi$ is not a proper vertex coloring. But, from Fact 3.1, any proper weighting from $\{1,2\}$ of $f(G)$ satisfies $\chi_{w}(v) \in\{4 n-2,4 n-1,4 n\}$ for all $v \in V$. Thus, there is no such proper weighting and hence $f(G) \notin 1-2$ WEIGHT.

This concludes the proof of Theorem 1.1

\section{Concluding remarks}

In this paper we showed that determining whether a graph has a proper weighting from either $\{0,1\}$ or $\{1,2\}$ is NP-complete. As a matter of fact, these two problems are not the same, in the sense that the corresponding families of graphs 0-1WEIGHT and 1-2WEIGHT are not equal. For example, the graph consisting only of one 2-edge gadget is in 1-2WEIGHT, as seen before, but it is easy to check that it is not in 0-1WEIGHT. Furthermore, we believe that our approach can be generalized to show that determining whether a graph has a proper weighting from $\{a, b\}$ is NP-complete for any different rational numbers $a$ and $b$. It is not clear if the same would hold for any two distinct irrational numbers.

\section{Acknowledgements}

We would like to thank Michał Karoński, who introduced us to the 1-2-3 conjecture. We are also very grateful to the referees for their detailed comments on an earlier version of this paper. 


\section{References}

L. Addario-Berry, K. Dalal, C. McDiarmid, B. A. Reed, and A. Thomason. Vertex-colouring edgeweightings. Combinatorica, 27(1):1-12, 2007. ISSN 0209-9683. doi: http://dx.doi.org/10.1007/ s00493-007-0041-6.

L. Addario-Berry, K. Dalal, and B. A. Reed. Degree constrained subgraphs. Discrete Appl. Math., 156(7):1168-1174, 2008. ISSN 0166-218X. doi: DOI:10.1016/j.dam.2007.05. 059. URL http://wWw. sciencedirect.com/science/article/B6TYW-4PT2FK1-1/ 2/f3371b3c9c874a200bf561073f921e12.

M. Kalkowski, M. Karoński, and F. Pfender. Vertex-coloring edge-weightings: Towards the 1-2-3conjecture. J. Comb. Theory, Ser. B, 100(3):347-349, 2010.

M. Karoński, T. Łuczak, and A. Thomason. Edge weights and vertex colours. J. Combin. Theory Ser. B, 91:151-157, 2004.

T. Wang and Q. Yu. On vertex-coloring 13-edge-weighting. Front. Math. China, 3(4):581-587, 2008. ISSN 1673-3452 (Print) 1673-3576 (Online). doi: 10.1007/s11464-008-0041-x. 